\title{
Experience with Robotic-assisted Microsurgery
}

\author{
Chih-Sheng Lai, MD *
}

Division of Plastic and Reconstructive Surgery, Department of Surgery, Taichung Veterans General Hospital, Taiwan, ROC

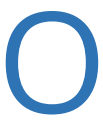

ver the past decade, Da Vinci Surgical System has made great strides in surgery. It has been widely applied in urology, gynecology, bariatric surgery, hepatobiliary surgery, thoracoscopic surgery, cardiac surgery, neurological surgery, and transoral otolaryngologic neoplasm resection. However, its application in plastic and reconstructive surgery still in the preliminary stages of development. I went to Hong Kong in April 2013 for the robotic surgical system training program and was awarded console surgeon certificate.

I started using the Da Vinci Surgical System since May 2013 and applied it to patients who needed free flap reconstruction after the oropharyngeal cancer resection. All the tumors resected without the lip-splitting and mandibulotomy, and the defects were reconstructed by free radial forearm fasciocutaneous flaps.

The microvascular anastomoses of the radial artery to the recipient artery, and one venae comitante to the recipient vein in the neck area were performed using a standard operating microscope. The anastomosis of another venae comitante to the recipient vein was performed ro-

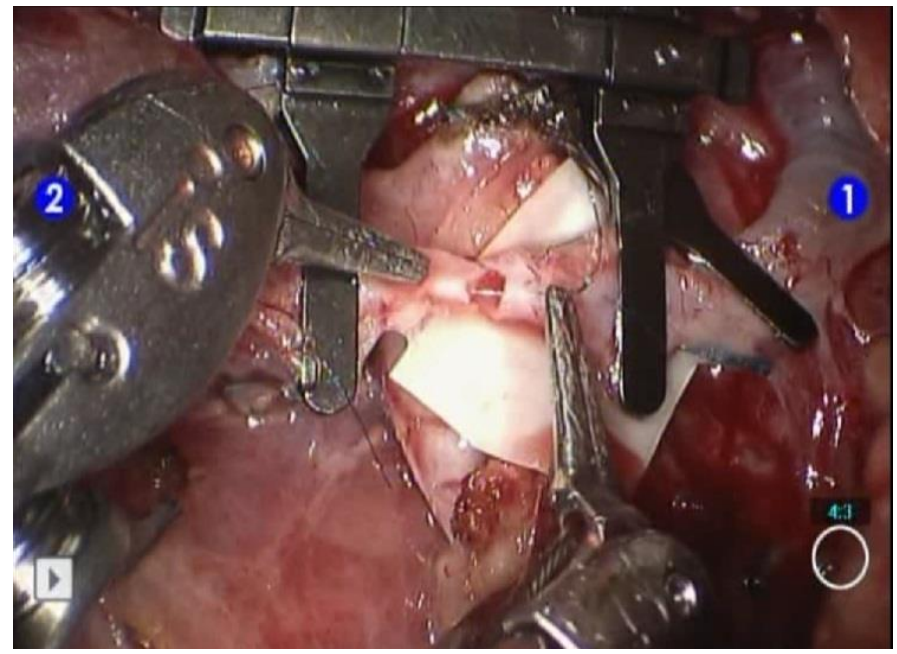

Figure 1. The anastomosis was performed robotically.

\section{${ }^{*}$ Correspondence: Chih-Sheng Lai, MD}

Division of Plastic and Reconstructive Surgery, Department of Surgery, Taichung Veterans General Hospital, Taiwan, ROC

E-mail: 01978626@pchome.com.tw

Received: June 5, 2017; Accepted: June 8, 2017; Published: July 9, 2017

International Microsurgery Journal. 2017;1(1):5

DOI:10.24983/scitemed.imj.2017.00022

Copyright (c) 2017 The Author. This is an open-access article distributed under the terms of the Creative Commons Attribution 4.0 International License (CC-BY). botically (Figure 1). Adequate microsurgery training is necessary to perform this procedure. I used visual cues to determine the tension while tying the knots. It is a crucial fact that a superior tactile sense of microsurgical manipulation is essential for this maneuver. The lack of haptic feedback when performing vascular anastomosis can be overcome by practicing visual cues. With the existing limited experience (1 artery and 9 veins), it is possible to perform microsurgical vascular suture using Da Vinci system, which achieves a patent and successful microvascular anastomosis. With the invention of finer devices, the standard operating microscope may be replaced by Da Vinci robotic surgical system.

Then, using 4-0 Monocryl sutures, which were manipulated by the Da Vinci system, the revascularized radial forearm flap was inset into the deepest portion of the oropharyngeal defect (Figure 2). Interrupted sutures were delivered by robotic arm-powered needle drivers (2 Black Diamond micro needle drivers, Intuitive Surgical). The Da Vinci Surgical System made it possible to reach difficult areas without using the jaw-splitting approach. Besides, it provided a high-resolution 3D stereoscopic view of the back of the mouth and throat.

In our study, we recruited 47 people who underwent reconstructive operations using a free radial forearm fasciocutaneous flap for oropharyngeal defects ( 14 robot-assisted and 33 conventional reconstructions). Our study revealed that there was no significant difference in complications or revision rates between the robot-assisted and conventional oropharyngeal reconstructions. The functional outcomes of robot-assisted reconstructions were superior to those of conventional reconstructions. The functional outcomes were assessed using the Functional Intraoral

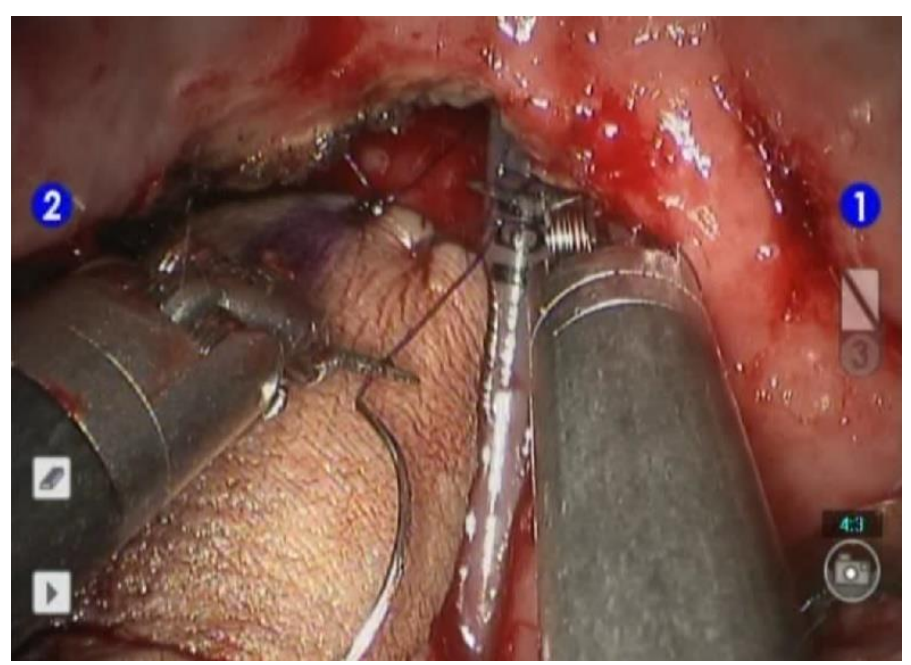

Figure 2. Inset of the flap into the oropharyngeal defect using the Da Vinci surgical system. 


\section{Editorial}

Glasgow Scale (FIGS), through which the function of the oral cavity after surgery (i.e., chewing, swallowing and speaking) was evaluated on a scale of 0 to 5 . The mean postoperative FIGS score was $10.29 \pm 2.02$ in the robot-assisted group and $8.42 \pm 2.29$ in the conventional group at 1 month postoperatively $(P=0.01)$. The mean postoperative FIGS score was 12.57 \pm 1.91 in the robot-assisted group and $9.91 \pm 3.09$ in the conventional group at 3 months post-operatively ( $P=0.005)$.

However, the drawback of the robotic surgery are robot space requirement, time-consuming installation, absence of force feedback, and the cost. However, compared with those of traditional techniques, the functional and aesthetic outcomes are superior. If the aforementioned drawbacks of the robotic system can be overcome and systematic learning of robotic techniques can be accomplished, the robotic surgical system may replace the microscope in microsurgery in the near future.

Minimal invasive surgery is the trend of the world of surgery. Da Vinci Surgical System has been extensively applied by many physicians in different fields of surgeries. However, in plastic and reconstructive surgery, it is limited to the need to create a new space in the human body in order to perform surgery, which does not bring about any significant progress in the development of minimal invasive surgery. Therefore, we need more accumulation of creativity and experience of the surgical technology to sustainable development. Perhaps in the near future, there will be considerable progress in the use of Da Vinci Surgical System in plastic surgery.

Da Vinci Surgical System is still unable to replace the surgeon for performing surgery. The robot is just a surgical instrument that assists the surgeon by providing a better surgical field of vision during surgery. The key to surgery is still the surgical skills and experience of the surgeon. Many endoscopic procedures, theoretically, can be replaced by the Da Vinci Surgical System to shorten the learning curve. As to whether a better outcome of surgery, it will be further confirmed in the future.

In December 2016, I attended Robotic Microsurgery Workshop in Nancy, France. This workshop was headed and implemented by Professor P. Liverneaux. There was dry-lab to practice vascular micro-anastomosis and cadaver-lab or pig-lab to practice flap harvesting. I will continue to bet on the robotic microsurgery. 